\title{
Anisotropic perception of visual angle: Implications for the horizontal-vertical illusion, overconstancy of size, and the moon illusion
}

\author{
ATSUKI HIGASHIYAMA \\ University of Osaka Prefecture, Osaka, Japan
}

\begin{abstract}
Three experiments investigated anisotropic perception of visual angle outdoors. In Experiment 1, scales for vertical and horizontal visual angles ranging from $20^{\circ}$ to $80^{\circ}$ were constructed with the method of angle production (in which the subject reproduced a visual angle with a protractor) and the method of distance production (in which the subject produced a visual angle by adjusting viewing distance). In Experiment 2, scales for vertical and horizontal visual angles of $5^{\circ}-30^{\circ}$ were constructed with the method of angle production and were compared with scales for orientation in the frontal plane. In Experiment 3, vertical and horizontal visual angles of $3^{\circ}-80^{\circ}$ were judged with the method of verbal estimation. The main results of the experiments were as follows: (1) The obtained angles for visual angle are described by a quadratic equation, $\theta^{\prime}=a+b \theta+c \theta^{2}$ (where $\theta$ is the visual angle; $\theta^{\prime}$, the obtained angle; $a, b$, and $c$, constants). (2) The linear coefficient $b$ is larger than unity and is steeper for vertical direction than for horizontal direction. (3) The quadratic coefficient $c$ is generally smaller than zero and is negatively larger for vertical direction than for horizontal direction. And (4) the obtained angle for visual angle is larger than that for orientation. From these results, it was possible to predict the horizontal-vertical illusion, overconstancy of size, and the moon illusion.
\end{abstract}

Under full-cue conditions, the apparent size of an object tends to remain constant, independently of the distance from which it is viewed. This phenomenon is called size constancy. For example, when the viewing distance of an object is doubled, the visual angle (or retinal image size) of the object decreases to about half the visual angle that the object subtends at the shorter distance, but the apparent size of the object at the longer distance is judged to be the same as the object's apparent size at the shorter distance.

Size constancy has often been explained by the perceptual interaction theory (Gogel, 1973a, 1973b) or by the apparent-distance theory (Rock, 1975), which assumes that the visual system transforms visual angle into apparent size by taking apparent distance into account. Thus, when the apparent distance of an object is shorter than the actual distance, the object should be perceived as being smaller than its actual size, as is often obtained under reduced distance-cue conditions (Gilinsky, 1955; Holway \& Boring, 1941; Ogasawara, 1933). On the other hand, when the apparent distance of an object is greater than the actual distance, the object should appear to be larger than its actual size. In either instance, according to the apparent-distance theory, the failure of attaining size constancy can be attributed to inaccurate distance judgments.

Although this explanation may be true in general, under some conditions the failure to attain size constancy may

Correspondence should be sent to Atsuki Higashiyama, Psychology Laboratory, University of Osaka Prefecture, Sakai, Osaka 591, Japan. be due to the inaccurate processing of visual angle rather than to the inaccurate processing of viewing distance. Some studies have shown that horizontal visual angle tends to be judged as being larger than the actual angle. Foley (1965), who used fractionation, magnitude estimation, and magnitude production to construct psychophysical scales for visual angle, described apparent visual angle as a linear function of actual angle, with a slope of 1.1 and an intercept of zero. Higashiyama (1984, 1987), obtaining absolute angle judgments in degrees, described the judged angle as a power function of the actual angle; that is, judged angle $=2.1 \times$ (actual angle $)^{0.9}$. This suggests that visual angle was overestimated by $30 \%$ on the average, and that the rate of overestimation is larger for smaller angles.

It appears that vertical visual angle is also overestimated. Kammann (1967) asked subjects to estimate a $34^{\circ}$ downhill slope and obtained mean estimates of $51^{\circ}$. Ross (1974) reported that less experienced skiers judged a $24^{\circ}$ uphill slope to be $47^{\circ}$, but that skillful skiers, when standing midway on the same slope, judged it to be $37^{\circ}$. Although other interpretations are possible, these results suggest that judged vertical visual angle was larger than actual angle.

It also appears that vertical visual angle is perceived as being larger than horizontal visual angle of the same magnitude. This anisotropic property of apparent visual angle can be predicted from studies of the horizontal-vertical illusion (e.g., Avery \& Day, 1969; Begelman \& Steinfeld, 1967; Chapanis \& Mankin, 1967; Künnapas, 1955; Morinaga, Noguchi, \& Ohishi, 1962; Ohno, 1972), which have shown that, when vertical and horizontal lines of the 
same physical length are viewed by an upright subject, the vertical length is perceived as being longer than the horizontal length. If apparent distance to the illusion figure is constant, the difference between the two apparent lengths may be due to the difference between the apparent visual angles. It follows that the vertical angle would be perceived as being larger than the horizontal angle.

The purpose of this study was to compare a scale for vertical visual angle with a scale for horizontal visual angle, and thereby to discover any implications for our understanding of the horizontal-vertical illusion, overconstancy of size, and the moon illusion. Three experiments are reported here. In Experiment 1, the subjects judged visual angles ranging from $20^{\circ}$ to $80^{\circ}$ by using both angle production and distance production. In the method of angle production, the subjects, standing erect away from a building, used a protractor to produce apparent visual angles subtended by two points on the the building's wall. In the method of distance production, the subjects determined their places by approaching the building or backing away from it, so that the visual angle formed by two points on the wall appeared equal to the standard angle designated by the protractor.

In Experiment 2, small visual angles of $5^{\circ}-30^{\circ}$, which were not examined in Experiment 1, were matched with the method of angle production. The scales for small visual angles were then compared with the scales for large visual angles obtained in Experiment 1 and with the scales for orientation from the reference axes in the frontal plane. In this case, the orientation condition was a control to show how large the visual angle was perceived to be.

In Experiment 3, the generality and robustness of the findings obtained with the protractor were examined with the method of verbal estimation, which does not entail a visual comparison of the standard with the variable. We asked the subjects to estimate visual angles in degrees over a full range of angles in a single session.

\section{EXPERIMENT 1}

\section{Method}

Subjects. The subjects were 18 undergraduates $(9$ males and 9 females). No special qualifying criteria were imposed.

Test field. The test field was an open space $(22 \times 32.5 \mathrm{~m})$ that was covered with orange concrete. There was a big tree at the center of the field, and there were always a few persons walking across the field. The shorter side of this field was adjacent to a three-story white building. Figure 1 shows the sketch of the building as seen by the subject. The wall of the building was flat, with a row of windows $(176 \mathrm{~cm}$ tall $\times 192 \mathrm{~cm}$ wide) on each floor.

Vertical and horizontal line lengths along the building's wall were used as standards. The vertical standard was a length of $11.15 \mathrm{~m}$, which was defined by the distance from the horizontal eye level to the roof of the building. To indicate the eye level of each subject, a piece of red vinyl tape was pasted on the vertical frame of a window on the first floor; the mean eye level was $155 \mathrm{~cm}$ from

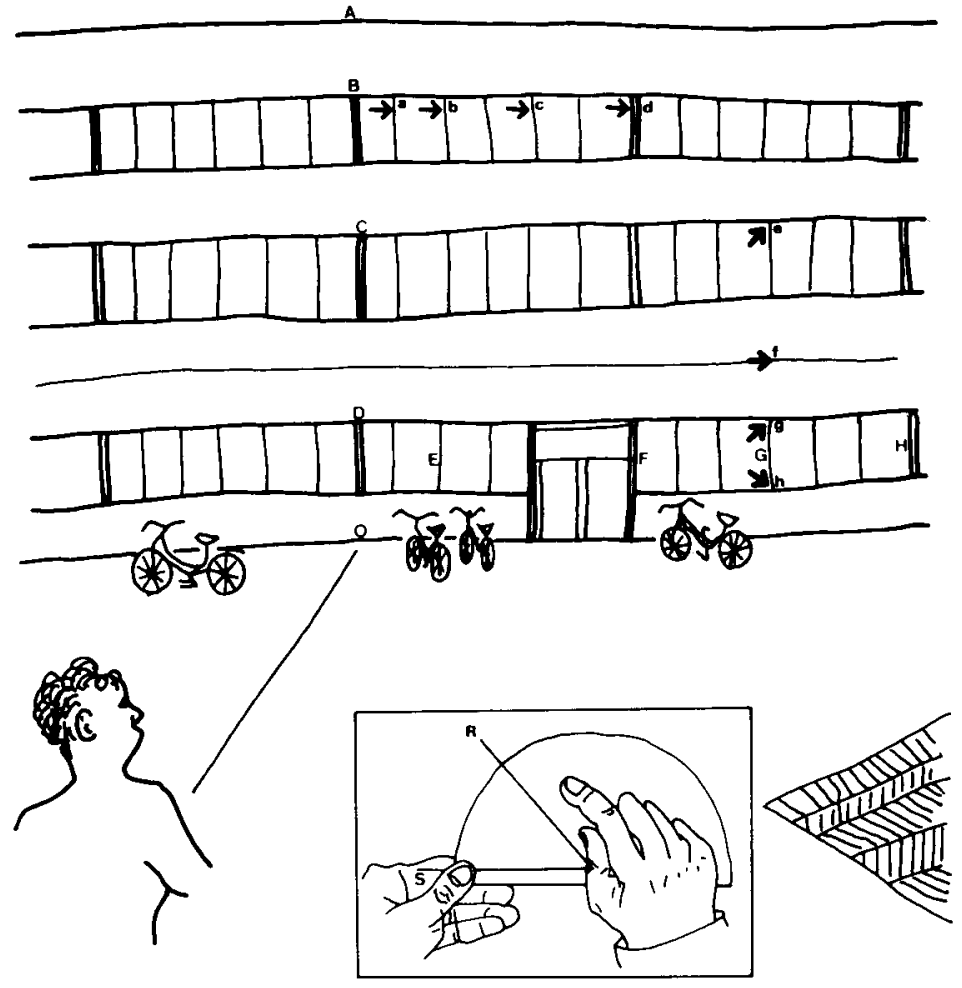

Figure 1. A sketch of the building and its surroundings as seen by the subject. See text for explanation of the letters $A-H$ and $a-h$. The inset at the bottom is a frontal view of the protractor with which the subject adjusted the angle between the stationary $\operatorname{rod}(S)$ and the rotatable $\operatorname{rod}(R)$. 
the ground. The horizontal standard was a length of $12.7 \mathrm{~m}$, which extended from the median plane toward the right. A piece of red tape was pasted at each end of the horizontal standard. The subject could easily identify the two standards from any position in the field without obstruction by the tree or persons.

Procedure. Two methods-angle production and distance production-were used to scale the visual angles. For each trial with the method of angle production, the subject was first asked to stand upright at a viewing distance of $3,6,10,14,20$, or $32 \mathrm{~m}$ from the building, and then to produce the visual angle subtended by each standard. The visual angles of the vertical (or horizontal) standard at the six viewing distances were $74.9^{\circ}\left(76.7^{\circ}\right), 61.7^{\circ}\left(64.7^{\circ}\right)$, $48.1^{\circ}\left(51.8^{\circ}\right), 38.5^{\circ}\left(42.2^{\circ}\right), 29.1^{\circ}\left(32.4^{\circ}\right)$, and $19.2^{\circ}\left(21.6^{\circ}\right) .^{1}$ The subject was not informed how far he or she was positioned from the building and was allowed to move his or her eyes and head freely.

A protractor was used to produce the apparent visual angle of the standard. It consisted of a semicircular transparent plastic plate ( $1 \mathrm{~mm}$ thick and $15 \mathrm{~cm}$ in diameter) and two thin steel rods $(15 \mathrm{~cm}$ long and $0.8 \mathrm{~mm}$ in diameter), which were installed at the center like the hands on a clock. One rod was rotatable around the center from $0^{\circ}$ to $180^{\circ}$, but the other was stationary at $0^{\circ}$.

The subject grasped the protractor throughout the experiment and was permitted to move it freely. However, when the subject adjusted the rotatable rod on the protractor, he or she was asked to hold the face of the protractor perpendicular to the line of sight, to keep the stationary rod oriented horizontally to the left, and not to direct the tips of the rods toward the standards. The inset in Figure 1 shows the protractor as viewed by the subject. The subject held the edge of the protractor with one hand and adjusted the rotatable rod with the other. Since the face of the protractor, on which the rods were installed, was covered with black paper, the subject could not read the protractor's scale. The experimenter, by turning the protractor over, read the scale values to an accuracy of $1^{\circ}$.

The main instructions to the subjects were:

Do you see the roof of the building and a red mark at your horizontal eye level (or do you see two red marks-one appearing straight ahead and another appearing to the right of it)? I think you realize that an angle is formed by the lines of direction from your eye to the marks. It is called a visual angle. We want to know the apparent extent of the visual angle. Please adjust the variable rod of the protractor to represent the angle you may perceive. In this adjustment, the stationary rod corresponds to the horizontal eye level (or the median plane), and the variable rod corresponds to the direction of the roof (or the right mark).

Since the subject made two angle adjustments, by extending (or rotating clockwise) and shrinking (or rotating counterclockwise), for each combination of viewing distance and standard, there were 24 (6 viewing distances $\times 2$ standards $\times 2$ rotation directions) trials for the method of angle production. The combinations of viewing distance and standard were presented to each subject in random order.

In each trial for the method of distance production, the subject was first allotted a standard angle on the protractor. The standard angles were $30^{\circ}, 40^{\circ}, 50^{\circ}, 60^{\circ}, 70^{\circ}$, or $80^{\circ}$. For each standard angle, the subject was asked to change his or her position in the open field, so that the visual angle of the standard line length appeared equal to the standard angle on the protractor. The standard angle on the protractor was set by the experimenter and given to the subject. The subject was not informed how large this standard angle actually was. The subject was allowed to move the protractor freely but, when looking at the standard angle, he or she was asked to hold the face of the protractor perpendicular to the line of sight, to keep the stationary rod oriented horizontally to the left, and not to direct the tips of the rods toward the standard line lengths. The experimenter moved with the subject to measure the distance to the subject from the building, to an accuracy of $10 \mathrm{~cm}$.

The main instructions to the subjects were:
Do you see the roof of the building and a red mark at your horizontal eye level (or do you see two red marks-one appearing straight ahead and another appearing to the right of it)? I think you realize that an angle is formed by the lines of direction from your eye to the marks. It is called a visual angle. We want to know the apparent size of the visual angle. Please walk back and forth until the visual angle appears equal to the standard angle on the protractor. In this protractor, the stationary rod corresponds to the horizontal eye level (or the median plane), and the variable rod corresponds to the direction of the roof (or the right mark).

Since the subject made two distance adjustments, by approaching the building and receding from the building, for each combination of standard angle and standard length, there were 24 (6 standard angles $\times 2$ standards $\times 2$ rotation directions) trials for the method of distance production. The combinations of standard line length and standard angle were presented to each subject in a random order.

Half the subjects participated first in the method of angle production and then in the method of distance production; the remaining subjects followed the reverse order.

\section{Results and Discussion}

Method of angle production. For the method of angle production, each subject provided a complete data set, but, for the method of distance production, 2 subjects failed to provide the complete sets of data. Therefore, separate analyses were done for the data obtained from the two methods.

Figure 2 shows mean angles obtained by the method of angle production as a function of visual angle of the standards. The parameter was the standard. Each data point was a mean taken across the 18 subjects and two

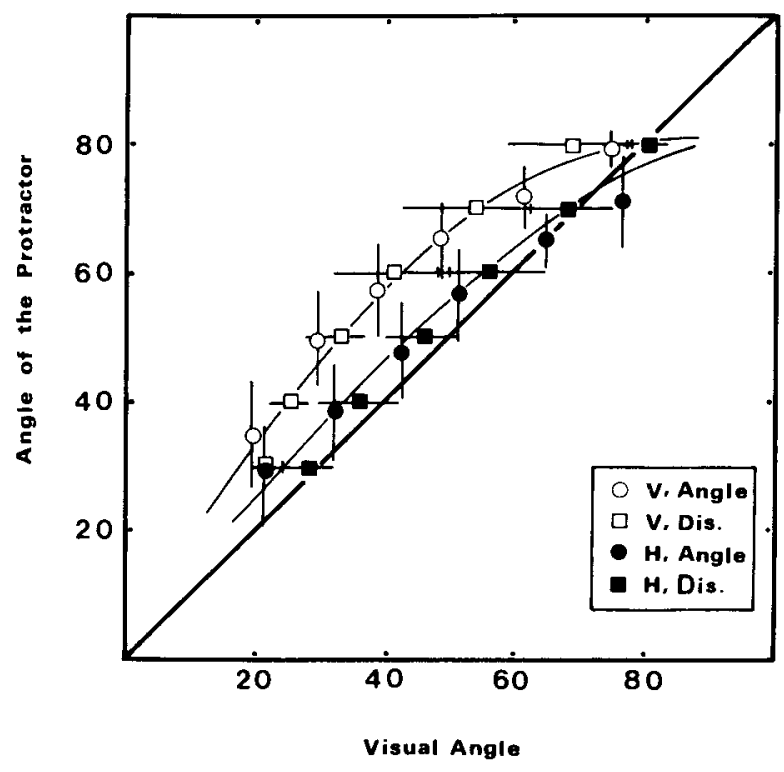

Figure 2. Matched angle (in degrees) of the protractor as a function of visual angle (in degrees). Open symbols stand for vertical visual angle and filled symbols stand for horizontal visual angle. Circles stand for the method of angle production, and squares stand for the method of distance production. The bars passing through the data points represent standard deviations. The upper and lower smooth curves are based on Equations 2 and 3, respectively. 
rotation directions. The matched angles were analyzed by a three-way analysis of variance (ANOVA) for repeated measures $(2$ standards $\times 6$ viewing distances $\times 2$ rotation directions). The main effect of size was significant $[F(1,17)=45.4, p<.001]$, indicating that the vertical angles were generally adjusted to be larger than were the horizontal angles.

The main effect of distance was also significant $[F(5,85)$ $=264.5, p<.001]$. A trend analysis, which was applied to mean angles as a function of standard angle (since the visual angles of the two standards at a viewing distance were slightly different, their mean was taken as the standard angle in this analysis), showed that the linear $[F(1,85)=1287.7, p<.001]$ and quadratic $[F(1,85)=$ $32.7, p<.001]$ components were significant and that the variation due to higher order trend components was not significant. The linear and quadratic components accounted for $97.4 \%$ and $2.5 \%$ of the variance, respectively.

The main effect of direction was significant $[F(1,17)=$ $17.0, p<.001]$. This suggests that the mean extending trial $\left(54.7^{\circ}\right)$ was smaller than the mean shrinking trial $\left(56.5^{\circ}\right)$. Two- and three-way interactions tested were not significant.

Method of distance production. Two subjects (1 male and 1 female) reported that they could not adjust distance for the standard angle of $30^{\circ}$, because the open field on which they walked back and forth was not large enough to match the small angle. Therefore, it was decided that the data obtained from the 2 subjects were excluded from the analysis for the method of distance production.

The distances adjusted by the 16 subjects were all transformed into visual angles that would be subtended by the standard when seen at the adjusted distances. For an adjusted distance of $x$ meters, the visual angle of the vertical standard equals $\tan ^{-1}(11.15 / x)$ and the visual angle of the horizontal standard equals $\tan ^{-1}(12.7 / x)$.

The visual angles thus transformed were shown in Figure 2 to compare with the results for the method of angle production. Note that the ordinate is the standard angle on the protractor and that the abscissa is the visual angle transformed from the adjusted distance. In other words, the ordinate is the independent variable, and the abscissa is the dependent variable. Each data point was a mean taken across the 16 subjects and two rotation directions. The parameter is the standard.

The transformed visual angles were analyzed by a threeway ANOVA for repeated measures ( 2 standards $\times 6$ standard angles $\times 2$ walking directions). The main effect of size was significant $[F(1,15)=59.1, p<.001]$, indicating that the transformed visual angles were generally smaller for the vertical standard than for the horizontal standard. This suggests that, when vertical angle is physically equal to horizontal angle, the vertical angle is perceived as being larger than the horizontal one.

The main effect of angle was significant $[F(5,75)=$ 408.3, $p<.001]$. A trend analysis, which was applied to the transformed visual angle as a function of standard angle, showed that the linear $[F(1,75)=2009.8, p<$
$.001]$ and quadratic $[F(1,75)=31.3, p<.001]$ components were significant and that the variation due to higher order trend components was not significant. The linear and quadratic components accounted for $98.4 \%$ and $1.5 \%$ of the variance, respectively. The main effect of direction was not significant. Tested two- and three-way interactions were not significant.

Scale for visual angle. A scale for visual angle was constructed for each combination of two methods and two standards. A multiple regression program was used to fit the following equation to the data points in Figure 2:

$$
\theta^{\prime}=a+b \theta+c \theta^{2},
$$

where $\theta$ stands for the visual angle, $\theta^{\prime}$ for the matched angle, and $a, b$, and $c$ for constants. ${ }^{2}$

Table 1 shows the estimates of $a, b$, and $c$ that were obtained by the method of least squares, together with the values of multiple correlation $(R)$. It is clear that Equation 1 provided an excellent description of visual angle judgments, showing that the values of multiple correlation were 0.997 or more.

A test of a subset of the parameters (Myers, 1966) showed that the quadratic coefficient $c$ for each equation in Table 1 was significantly different from zero $[F(1,3)>$ $11.7, p<.05$ ], after the variabilities predictable from linear term $b$ had been removed. This means that the matched angle is a negatively accelerated, rather than linear, function of visual angle, consistent with the results of the analyses of trend mentioned above.

By averaging the two estimates for each of the three parameters $a, b$, and $c$ in Table 1, we constructed the scale for the vertical,

$$
\theta_{v}^{\prime}(\theta)=0.52+1.86 \theta-0.0107 \theta^{2},
$$

and the scale for the horizontal,

$$
\theta_{\mathrm{h}}^{\prime}(\theta)=-3.18+1.35 \theta-0.0047 \theta^{2} \text {, }
$$

where $20^{\circ} \leq \theta \leq 80^{\circ}$.

The two curves in Figure 2 are based on Equations 2 and 3. It can be seen that the matches for horizontal visual angles were generally larger than the actual visual angles. This result is in good agreement with the studies of Foley (1965) and Higashiyama $(1984,1987)$. It is also clear that the vertical visual angle is adjusted to be larger than the horizontal visual angle of the same size. This appears to be a new finding.

Table 1

\begin{tabular}{|c|c|c|c|c|}
\hline Method & $a$ & $b$ & $c$ & $R$ \\
\hline \multicolumn{5}{|c|}{ Vertical } \\
\hline $\begin{array}{l}\text { Distance production } \\
\text { Angle production }\end{array}$ & $\begin{array}{r}-5.94 \\
6.97\end{array}$ & $\begin{array}{l}2.04 \\
1.68\end{array}$ & $\begin{array}{l}-0.0117 \\
-0.0097\end{array}$ & $\begin{array}{l}0.997 \\
0.997\end{array}$ \\
\hline \multicolumn{5}{|c|}{ Horizontal } \\
\hline $\begin{array}{l}\text { Distance production } \\
\text { Angle production }\end{array}$ & $\begin{array}{r}-6.14 \\
2.96 \\
\end{array}$ & $\begin{array}{l}1.40 \\
1.30 \\
\end{array}$ & $\begin{array}{l}-0.0042 \\
-0.0052\end{array}$ & $\begin{array}{l}0.999 \\
0.999 \\
\end{array}$ \\
\hline
\end{tabular}

Estimates of the Parameters Involved in the Quadratic Equation $\theta^{\prime}=a+b \theta+c \theta^{f}$ and the Multiple Correlations $R$ 


\section{EXPERIMENT 2}

Since Experiment 1 did not provide direct evidence as to the matches of visual angles of less than $20^{\circ}$, one may attempt to infer them by applying Equations 2 and 3 to such small visual angles. However, this attempt may not be successful. These equations, for example, would predict that the vertical and horizontal visual angles of $5^{\circ}$ would be adjusted at $9.6^{\circ}$ and $3.5^{\circ}$, respectively. These predictions seem unlikely, because studies of the horizontal-vertical illusion predict that the vertical visual angle will not be adjusted to twice the size of the horizontal visual angle.

In Experiment 2, therefore, our goal was to examine whether the trends found in Experiment 1 would be true for visual angles of $5^{\circ}-30^{\circ}$. Using the method of angle production, the subjects judged visual angles while the viewing distance was held constant.

One might also argue that the trend of overestimating the visual angle in Experiment 1 reflects a judgmental bias due to the use of the protractor and does not reflect any intrinsic property of angle perception. To rule out this possibility, the matches for visual angle were compared with those for orientation from the reference axes in the frontal plane. If matching with the protractor led the subjects to generate angles larger than actual angles, angles of orientation would be adjusted exactly as much as visual angles.

\section{Method}

Subjects. The subjects were 16 undergraduates (10 males and 6 females), none of whom served in Experiment 1. No special qualifying criteria were imposed.

Test field. The test field was the same as that used in Experiment 1 . The subject viewed the building from a distance of $30 \mathrm{~m}$. The vertical lengths were defined as distances from the reference ground $(O)$ to the salient marks on the building wall. We used as marks the roof of the building and three upper sides of the window frames, as indicated by the letters A, B, C, and D in Figure 1. Since these marks were $2.7,6.7,10.5$, and $12.7 \mathrm{~m}$ distant from the reference ground, the visual angles of these vertical lengths were $5.1^{\circ}$, $12.6^{\circ}, 19.3^{\circ}$, and $22.3^{\circ}$, respectively. The horizontal standard lengths were defined as distances from the median plane to four lateral sides of the window frames at the first floor, as indicated by the letters $E, F, G$, and $H$ in Figure 1 . A piece of red vinyl tape was pasted on each of these sides. Since these lateral sides were 2, 6, 9, and $14 \mathrm{~m}$ apart from the median plane, the visual angles of these horizontal lengths were $3.8^{\circ}, 11.3^{\circ}, 16.7^{\circ}$, and $25.0^{\circ}$, respectively.

To generate orientation in the frontal plane, eight orange arrows ( $54 \mathrm{~cm}$ long $\times 39 \mathrm{~cm}$ wide) were pasted on the building wall. Four arrows were placed on the windows on the third floor, as shown by the letters $a, b, c$, and $d$ in Figure 1. Since the heads of the arrows were $1,2,4$, and $6 \mathrm{~m}$ apart from the median plane and $10.5 \mathrm{~m}$ high from the ground, the imaginary lines connecting these arrowheads with the reference ground intersected the median plane at angles of $5.4^{\circ}, 10.8^{\circ}, 20.9^{\circ}$, and $29.7^{\circ}$, respectively. We refer to these angles as orientations from the vertical axis. Four other arrows were arranged vertically at the lateral distance of $9 \mathrm{~m}$ from the median plane, as indicated by the letters $e, f, g$, and $h$ in Figure 1 . Since these arrowheads were $1.1,2.7,4.0$, and $6.7 \mathrm{~m}$ high from the ground, the imaginary lines connecting them with the reference ground were $7.0^{\circ}, 16.7^{\circ}, 24.0^{\circ}$, and $36.7^{\circ}$ apart from the base of the building, respectively. We refer to these angles as orientations from the horizontal axis.

Procedure. Each subject was asked to see the building wall at a viewing distance of $30 \mathrm{~m}$ and to ensure the positions of the refer- ence ground, the reference axes, the roof, the window frames, and the arrows that were used to define visual angles and orientations. After receiving the instructions, which were similar to the instructions for the method of angle production in Experiment 1, the subject was asked to produce the visual angles and the orientation angles with the protractor. The subject was required to stand upright throughout the experiment but was allowed to move the eyes and head freely.

Since the subject made two angle adjustments, by extending and shrinking, for each standard angle, there were 32 trials [ 8 visual angles +8 orientations) $\times 2$ rotation directions]. Half the subjects first attempted matches of visual angle and then matches of orientation; the remaining subjects followed the reverse order. In each task, the order for the directions of visual angle (or reference axis) was counterbalanced among the subjects.

\section{Results and Discussion}

Short-range analyses. Figure 3 shows the mean angle obtained with the protractor as a function of orientation or visual angle. Each data point was a mean taken across the 16 subjects and two rotation directions. The parameter was the direction of the visual angle and the reference axis (i.e., vertical visual angle, horizontal visual angle, orientation from the vertical axis, and orientation from the horizontal axis).

Equation 1 was fitted to each of the four sets of data points in Figure 3. The results showed that for any equation, the quadratic coefficient was not significantly different from zero after the variabilities predictable from the linear term had been removed. This means that, for the angle range employed in Experiment 2, the matched angle is parsimoniously described by a linear function of actual angle.

According to a least squares criterion, a linear function was individually fitted to each of the four sets of

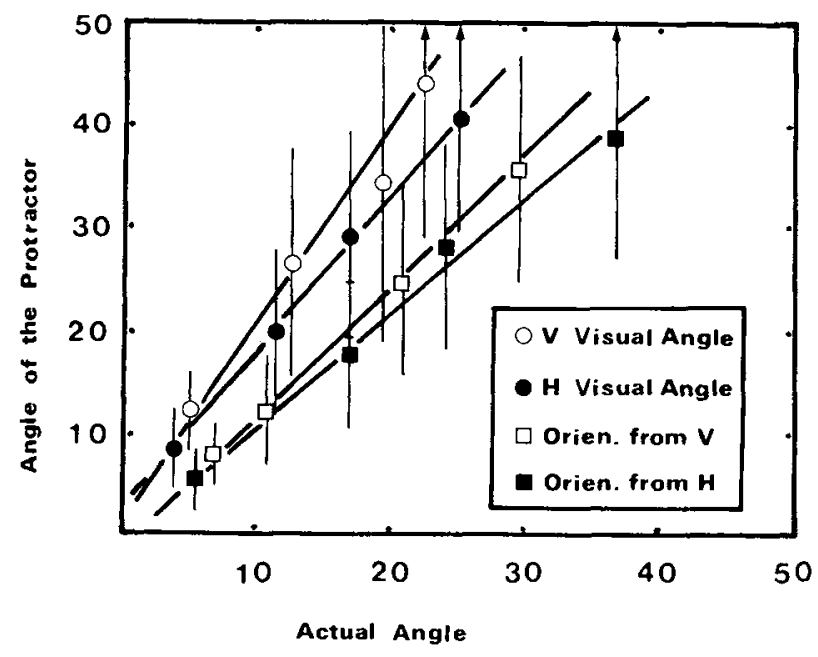

Figure 3. Matched angle of the protractor (in degrees) as a function of stimulus angle (in degrees). Open circles stand for the vertical visual angle; filled circles stand for the horizontal visual angle; open squares stand for the orientation from the vertical axis; filled squares stand for the orientation from the horizontal axis. The bars passing through the data points represent standard deviations. The slope and intercept of the best-fitting linear function in each condition are shown in Table 2. 
Table 2

Mean Slopes and Intercepts of Linear Equations Fitted to Obtained Angle and Mean Coefficient of Determination $r^{2}$ : The Parameter Is the Direction of Visual Angle and Reference Axis

\begin{tabular}{llll}
\hline Direction & Slope & Intercept & $r^{2}$ \\
\hline \multicolumn{4}{c}{ Visual Angle } \\
Vertical & 1.89 & 2.46 & \\
Horizontal & 1.54 & 2.77 & 0.965 \\
& Orientation & & 0.976 \\
Vertical & 1.29 & -1.25 & \\
Horizontal & 1.14 & -0.32 & 0.987 \\
\hline
\end{tabular}

matched angles. Table 2 shows mean slopes, intercepts, and $r^{2}$, averaged over the 16 subjects. The means of the best-fitting functions are drawn in Figure $3 .^{3}$

A one-way repeated measures ANOVA done on the slope data showed that the main effect of direction was significant $[F(3,45)=16.37, p<.001]$. A contrast analysis (Rosenthal \& Rosnow, 1985) showed that (1) the slope was significantly steeper for the vertical than for the horizontal visual angle $[F(1,45)=9.59, p<.01],(2)$ there was no significant difference between the two orientation conditions $(F=1.78)$, and (3) the combination of the two visual angle conditions $(M=1.71)$ was significantly steeper than the combination of the two orientation conditions $(M=1.21)$.

A one-way repeated measures ANOVA done on the intercept data showed that the main effect of direction was significant $[F(3,45)=6.19, p<.01]$. A contrast analysis showed that (1) the intercepts were not significantly different between the two visual angle conditions $(F<1)$ or between the two orientation conditions $(F<1)$, and (2) the combination of the two visual angle conditions $(M=2.62)$ was significantly larger than the combination of the two orientation conditions $(M=-0.78)$. For the horizontal visual angle condition, the intercept was significantly different from zero $[t(15)=2.65, p<.05]$, but this was not the case for each of the three other conditions.

It is clear from Figure 3 and Table 2 that the slopes for visual angle were larger than those for orientation, and that the slope for vertical visual angle was 23\% steeper than the slope for horizontal visual angle, comparable with the linear coefficients obtained in Experiment 1 (i.e., 1.86 for the vertical and $\mathbf{1 . 3 5}$ for the horizontal).

Full-range analyses. To compare with the results of Experiment 1, the matches obtained for visual angle in Experiment 2 were superimposed on Figure 2. Figure 4 is the result. Equation 1 was fitted to the matched angles for each of the vertical and horizontal directions. The results are that for the vertical,

$$
\theta_{\mathrm{v}}^{\prime}(\theta)=4.78+1.71 \theta-0.0095 \theta^{2}
$$

and for the horizontal,

$$
\theta_{\mathrm{h}}^{\prime}(\theta)=8.11+1.03 \theta-0.0022 \theta^{2},
$$

where $4^{\circ} \leq \theta \leq 80^{\circ}$. Multiple regression was 0.991 for Equation 4 and 0.987 for Equation 5. These very high values suggest that the results of the two experiments are internally consistent, despite the different subjects and sessions. The two smooth curves in Figure 4 are based on Equations 4 and 5.

Let us consider how well Equations 4 and 5 predict matches for small visual angles. For example, the vertical angle of $5^{\circ}$ is predicted to be matched to $13.1^{\circ}$, whereas the horizontal angle of the same magnitude is predicted to be matched to $13.2^{\circ}$. This suggests that the matches for small angles would not differ between the two directions. On the other hand, from the linear functions shown in Table 2, we obtain somewhat different predictions. For the vertical and horizontal visual angles of $5^{\circ}$, the matches are predicted to be $11.9^{\circ}$ and $10.5^{\circ}$, respectively. In other words, the matches for the vertical angle are $17 \%$ larger than those for the horizontal angle. The difference may be mainly due to the number of data points that were used to fit each equation: 16 for the quadratic function, and 4 for the linear function.

In predicting vertical and horizontal matches for small visual angles, the quadratic function may be more desirable than the linear function, because the matches predicted from the quadratic functions may be compatible with studies of the horizontal-vertical illusion in the laboratory. Since the amount of the horizontal-vertical illusion varies, depending on the test figure used (Künnapas, 1955; Morinaga et al., 1962), the orientation of the head (Avery \& Day, 1969; Morinaga et al., 1962; Ohno, 1972), the standard line length (Begelman \& Steinfeld, 1967), the assignment of the standard and comparison (Begelman \& Steinfeld, 1967), and so forth, it is difficult to predict the exact amount of illusion for small visual angles, but it may be about $3 \%-4 \%$ on the average, wavering between $-1 \%$ and $12 \%$. In particular, in well-illuminated conditions, no study that we have surveyed has yielded an illusion of $10 \%$ or more.

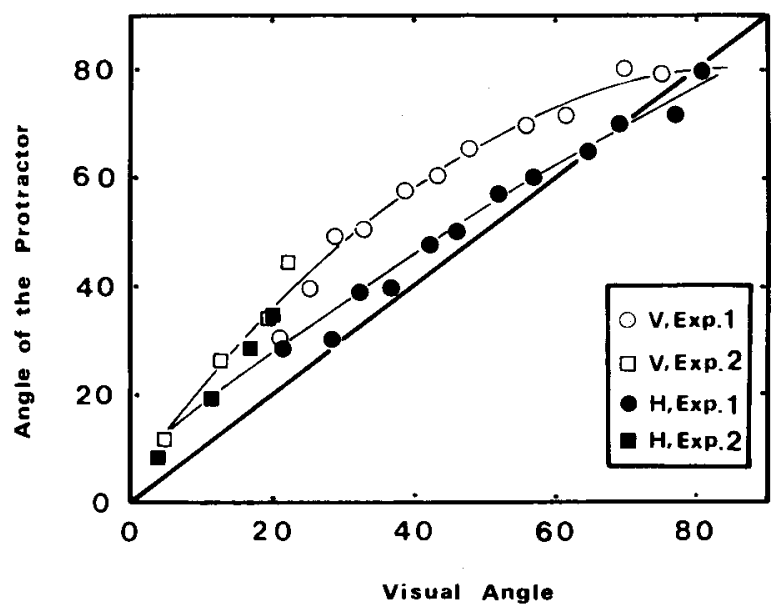

Figure 4. The results of Experiment 2, superimposed on Figure 2. Open symbols stand for vertical visual angle; flled symbols stand for horizontal visual angle. The upper and lower smooth curves are based on Equations 4 and 5, respectively. 
Despite this successful fit of the quadratic function to the data, one may want to describe the same data by the power function with two free parameters $\left(\theta^{\prime}=k \theta^{p}\right)$, because the power function assumes $\theta^{\prime}$ of zero for a $\theta$ of zero. Since the power function predicts a straight line between matched angle and visual angle on log-log coordinates, slope $(p)$ and intercept $(\log k)$ were estimated, with a least squares criterion, for each of the directions. The obtained parameters were, for the vertical, $k=4.80$, $p=0.639$, and $r^{2}=.925$, and for the horizontal, $k=$ $3.50, a=0.701$, and $r^{2}=.974$. It seems that the power function provides as good a fit to the data as does the quadratic function. However, the power function may not be desirable for matches predicted for small visual angles. For example, a vertical visual angle of $5^{\circ}$ is predicted to be matched to $13.4^{\circ}$, whereas a horizontal angle of the same size is predicted to be matched to $10.8^{\circ}$. This means that the vertical angle would be perceived as $24 \%$ larger than the horizontal angle. This overperception of vertical visual angle seems too large in the light of previous literature on the horizontal-vertical illusion.

Comparison of visual angle and orientation. To clarify how large the visual angle appears, it seems appropriate to compare it with the matches for orientation. Table 2 shows that orientation, on the average, was adjusted as being $21 \%$ larger than it actually was. This deviation does not differ greatly from the results of other studies. Lennie (1971), asking subjects to reproduce a $40^{\circ}$ standard by the variables that were oriented differently from the standard, found that the matches were constantly larger than the standard, with a maximum of $6^{\circ}$ for the variable being oriented obliquely. Dick and Hochstein (1989) found that the orientation from the horizontal axis was overestimated but that the orientation from the vertical axis was underestimated, and that the maximal deviation of $6^{\circ}$ was obtained for an orientation of $30^{\circ}$ from each reference axis. These results suggest that orientation is not judged precisely, in particular, for oblique orientation.

It seems that the overmatches of $21 \%$ obtained for orientation judgments in this study include at least two components-one due to physiological distortion in perceiving orientation, and another due to judgmental bias in matching angles with the protractor. It follows that the component due only to the judgmental bias may be less than $21 \%$. Therefore, if the matches for visual angle were based only on judgmental bias, the slope for visual angle would not be over 1.21. However, the obtained slope was 1.71. This implies that, even if the matches for visual angle are contaminated by the judgmental bias, its steep slope cannot be explained exclusively by the judgmental bias.

\section{EXPERIMENT 3}

Despite the evidence obtained in Experiments 1 and 2, we attempted to measure apparent visual angles with a method that was free from the orientation of the protractor, because matches for visual angle may depend on orientation of the protractor (e.g., Nakatani, 1991). In
Experiment 3, therefore, we asked the subjects to estimate visual angles verbally over a full range of angles in a single session.

\section{Method}

Subjects. The subjects were 20 undergraduates and teaching staff (14 males and 6 females), none of whom served in Experiments 1 and 2 . No special qualifying criteria were imposed.

Test field. The test field was the same as that in Experiment 1. The standard line lengths were the same as those in Experiment 2, except that, first, the subject viewed each standard from the distances of 3,10 , and $30 \mathrm{~m}$, and, second, the lower end point of the vertical standards was the eye level of each subject, as used in Experiment 1 . The eye level was indicated by pasting up a piece of red tape on a window frame on the first floor. The mean eye level was $155 \mathrm{~cm}$ high. Hence, the visual angles of the vertical standards were $74.9^{\circ}, 71.5^{\circ}, 59.6^{\circ}$, and $21.0^{\circ}$ at a viewing distance of $3 \mathrm{~m}$, $48.1^{\circ}, 41.8^{\circ}, 27.2^{\circ}$, and $6.6^{\circ}$ at a viewing distance of $10 \mathrm{~m}$, and $20.4^{\circ}, 16.6^{\circ}, 10.4^{\circ}$, and $2.2^{\circ}$ at a viewing distance of $30 \mathrm{~m}$. The visual angles of the horizontal standards, on the other hand, were $77.9^{\circ}, 71.6^{\circ}, 63.4^{\circ}$, and $33.7^{\circ}$ at a viewing distance of $3 \mathrm{~m}, 54.5^{\circ}$, $42.0^{\circ}, 31.0^{\circ}$, and $11.3^{\circ}$ at $10 \mathrm{~m}$, and $25.0^{\circ}, 16.7^{\circ}, 11.3^{\circ}$, and $3.8^{\circ}$ at $30 \mathrm{~m}$.

Procedure. One half of the group of subjects were asked to stand upright at each of the three viewing distances and to verbally estimate the apparent visual angles of each of the four vertical standards in degrees. The order of viewing distances was randomly determined for each subject, and, for each viewing distance, the order of the four visual angles was also randomly determined. After completing estimations for the 12 vertical angles ( 4 standards $\times 3$ viewing distances), the subjects estimated horizontal angles in the same manner as they estimated the vertical angles. The remaining half of the subjects estimated the horizontal angles first and the vertical angles second.

The main instructions for the vertical angles were:

Do you see the roof of the building and the upper window frames on the first, second, and third floors? We call these positions the test points. Do you also see a red mark at your horizontal eye level? I want to know a visual angle by which each of the test points is separated vertically from the eye level. Please tell me how large the visual angle appears by reporting numbers in degrees.

The instructions for horizontal angle were similar to those for vertical angle.

\section{Results and Discussion}

Figure 5 shows mean estimates as a function of viewing distance, with the parameters of standard size and direction. Each data point was a mean taken across the 20 subjects. A two-way ANOVA for repeated measures (3 viewing distances $\times 4$ standards) was performed for each direction. For the vertical, the main effects of distance $[F(2,38)=205.5, p<.001]$ and size $[F(3,57)=$ $435.9, p<.001]$ were significant, and the distance $\times$ size interaction was significant $[F(6,114)=18.6, p<$ $.001]$. For the horizontal, the main effects of distance $[F(2,38)=70.1, p<.001]$ and size $[F(3,57)=499.7$, $p<.001]$ were significant, and the distance $\times$ size interaction was significant $[F(6,114)=7.0, p<.001]$.

The two significant interactions suggest that the difference between the largest and the smallest estimates decreased with an increase in viewing distance. This result may reflect the fact that the difference between the largest and the smallest visual angles diminishes with viewing 

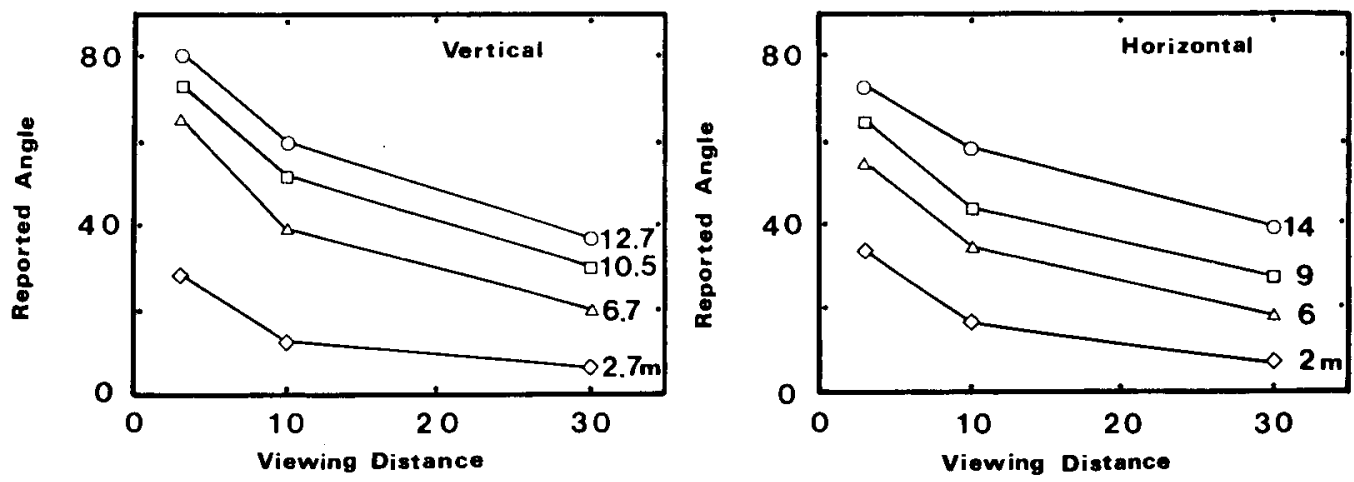

Figure 5. Reported angle (in degrees) as a function of viewing distance (in meters), with the parameter of standard size (in meters). The left panel shows the results for the vertical, and the right panel shows the results for the horizontal.

distance. (For example, the difference between the largest and smallest vertical standards was $53.9^{\circ}$ at a viewing distance of $3 \mathrm{~m}$, but it was reduced to $18.2^{\circ}$ at a viewing distance of $30 \mathrm{~m}$.)

Figure 6 shows mean estimates as a function of visual angle, with the parameters of standard direction and viewing distance. Equation 1 was fitted to the data points of each direction. For the vertical,

$$
\theta_{\mathrm{v}}^{\prime}(\theta)=5.27+1.40 \theta-0.0059 \theta^{2},
$$

and for the horizontal,

$$
\theta_{\mathrm{h}}^{\prime}(\theta)=7.17+1.04 \theta-0.0032 \theta^{2},
$$

where $3^{\circ} \leq \theta \leq 78^{\circ}$. Multiple correlations were .993 for Equation 6 and .978 for Equation 7. The two smooth curves in Figure 6 are based on Equations 6 and 7.

The quadratic coefficient in Equation 6 was significantly different from zero $[F(1,9)=8.23, p<.05]$, after the

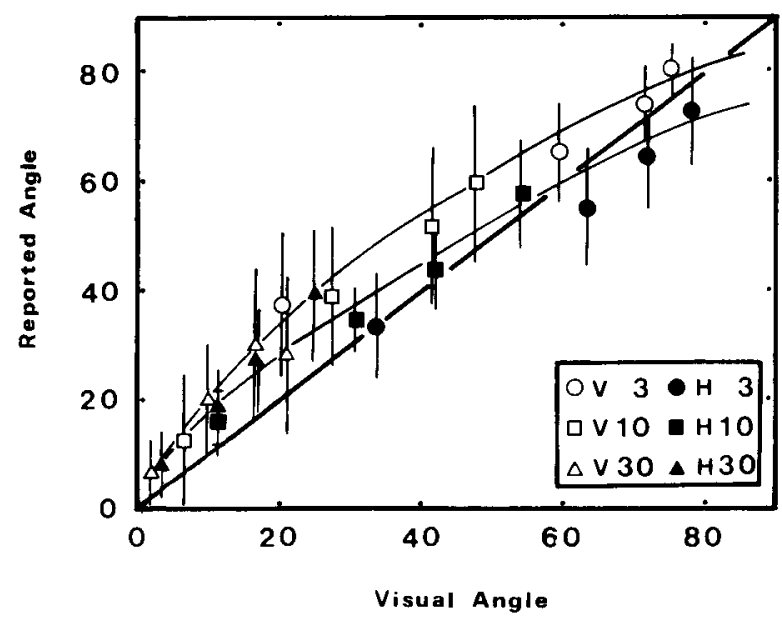

Figure 6. Reported angle (in degrees) as a function of visual angle (in degrees). Open circles stand for the vertical angle; filled circles stand for the horizontal angle. The bars passing through the data points represent standard deviations. The upper and lower smooth curves are based on Equations 6 and 7, respectively. variability predictable from the linear term had been removed, but the quadratic coefficient in Equation 7 was not. Thus, the estimates for vertical angle are a negatively accelerated function of visual angle, whereas the estimates for horizontal angle are a linear function of visual angle.

Equation 1 was fitted individually to each of the vertical and horizontal sets of estimates. The difference between the two linear coefficients was significant $[t(19)=$ 2.37, $p<.05$ ], but the difference between the two constant coefficients and the difference between the two quadratic coefficients were not significant. The constant coefficient was significantly different from zero for the vertical $[t(19)=2.56, p<.05]$ and horizontal $[t(19)=$ $3.48, p<.01]$ directions.

Equations 6 and 7 may predict estimates for small visual angles satisfactorily. For example, the vertical and horizontal visual angles of $5^{\circ}$ are predicted to be estimates of $12.1^{\circ}$ and $12.3^{\circ}$, respectively. This suggests that there is little or no difference in estimates between the two directions for small visual angles.

The data points in Figure 6 were also fitted by the power function $\left(\theta^{\prime}=k \theta^{p}\right)$. The obtained parameters were, for the vertical, $k=3.67, p=.713$, and $r^{2}=0.986$, and for the horizontal, $k=3.29, a=0.703$, and $r^{2}=.972$. This suggests that the power function provides as good a fit to the data as does the quadratic function. However, the ratio of vertical to horizontal estimates for small visual angles was much larger than the amount of the horizontalvertical illusion (3\%-4\%). For example, for a visual angle of $5^{\circ}$, the ratio of vertical to horizontal estimates is about 1.14 , since the vertical and horizontal estimates are predicted to be $11.6^{\circ}$ and $10.2^{\circ}$, respectively.

\section{GENERAL DISCUSSION}

The results of the three experiments can be summarized as follows. First, when visual angle was adjusted with the protractor or was estimated verbally, the obtained angles were described reasonably well by Equation 1 . Second, the linear coefficient in Equation 1 was generally larger than unity and was steeper for the vertical than for the 


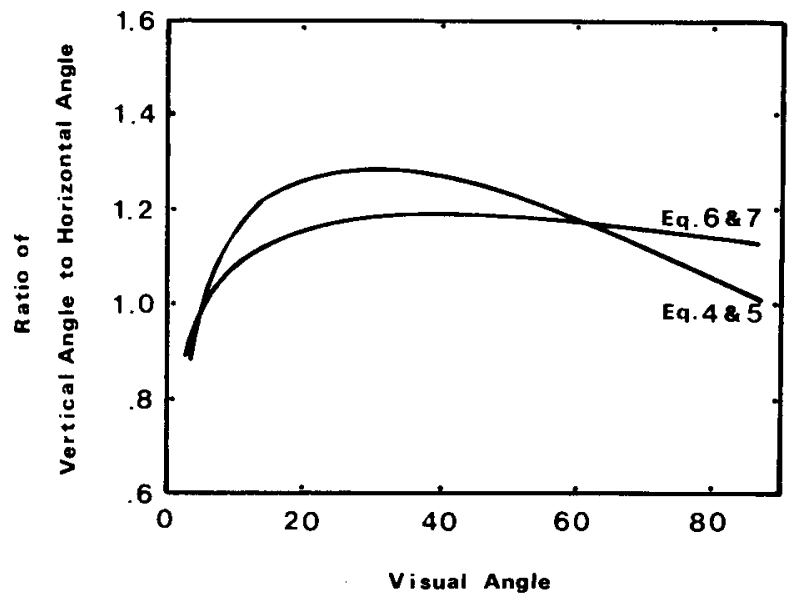

Figure 7. The ratio of vertical to horizontal matches, computed from Equations 4 and 5, and the ratio of vertical to horizontal estimates, computed from Equations 6 and 7. The ordinate represents the ratio, and the abscissa represents the visual angle (in degrees).

horizontal. Third, the quadratic coefficient in Equation 1 was generally smaller than zero, and its value for the vertical was negatively larger than that for the horizontal. This suggests that the obtained angle does not grow in proportion to the visual angle, implying that visual angle is not processed precisely. Fourth, a visual angle was judged larger than the angle of the same magnitude in the frontal plane. Taken together, the second and fourth findings suggest that the vertical visual angle appears largest, the frontal-plane angle appears smallest, and the horizontal visual angle appears to be intermediate between the two.

These findings provide an explanation for some perceptual riddles - the horizontal-vertical illusion, overconstancy of size, and the moon illusion, which may be difficult to explain as long as we assume that apparent visual angle is processed in proportion to actual visual angle.

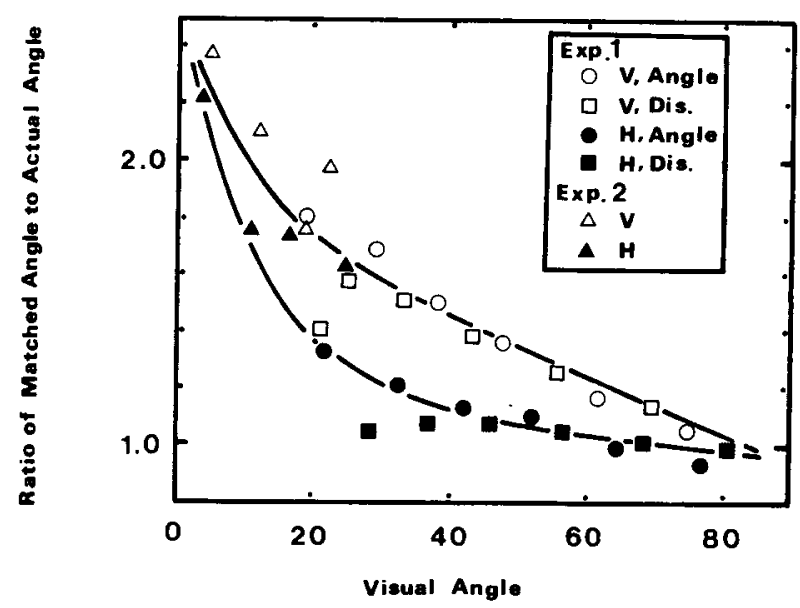

\section{The Horizontal-Vertical Illusion}

Figure 7 shows the ratio of vertical to horizontal matches (and estimates) as a function of visual angle. Each curve in Figure 7 is drawn on the basis of each pair of equations: Equations 4 and 5 and Equations 6 and 7 . The two curves are similar to each other, despite the different subjects and methods of measurement. The ratio is smallest for small visual angles, and it increases as visual angle increases, reaches a maximum at $30^{\circ}-60^{\circ}$, and then decreases as visual angle increases further. In other words, for a small visual angle of $10^{\circ}$ or less, the vertical angle was judged, at best, as being $10 \%$ larger than the horizontal angle, whereas for a medium visual angle, the vertical angle was judged as being $10 \%-30 \%$ larger than the horizontal angle. ${ }^{4}$

A comparison between previous studies of the horizontalvertical illusion suggests that a large amount of illusion is more likely to be obtained with big objects in an outdoor setting than with small L- and inverted-T-shaped figures in the laboratory. Chapanis and Mankin (1967) measured the amount of the horizontal-vertical illusion for each of 10 different objects that had different sizes but that subtended the same visual angle $\left(22^{\circ}\right)$ in naturalistic situations. According to computations that we did with their data, the mean amount of illusion was $14.4 \%$ for five large objects $3.9-18.3 \mathrm{~m}$ tall and $3.0 \%$ for five small objects 7.6-135 cm tall. Higashiyama (1991) had the subject approach or back away from a building, so that the distance to the building from the subject appeared to be equal to the vertical and horizontal standard line lengths (3-14 $\mathrm{m}$ ) along the building wall. The mean distance for the vertical standard is $21 \%-35 \%$ larger than that for the horizontal standard.

\section{Overconstancy of Size}

Figure 8 shows the ratio of obtained angle to visual angle as a function of visual angle, with the parameter of

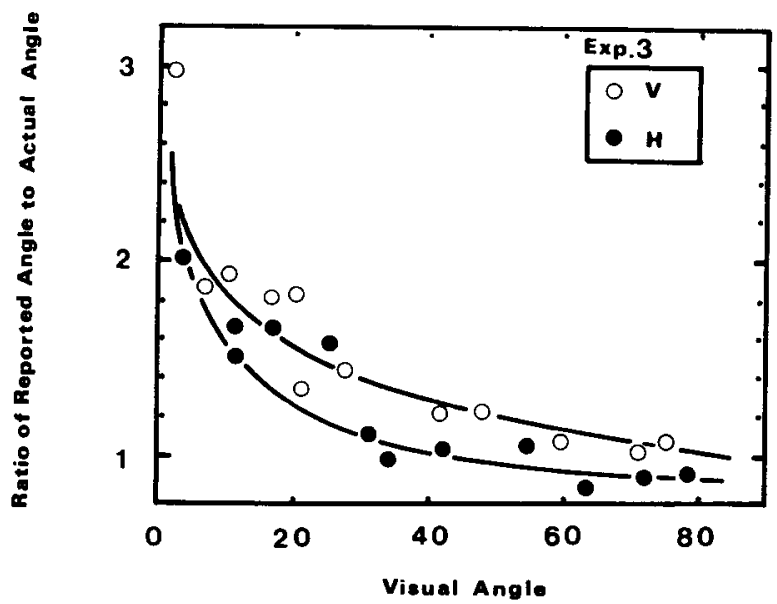

Figure 8. The ratio of obtained angle to visual angle as a function of actual visual angle (in degrees). The left panel shows the results obtained with the protractor (Experiments 1 and 2), and the right panel shows the results obtained with verbal estimation (Experiment 3). Open symbols stand for vertical visual angle; filled symbols stand for horizontal visual angle. The upper and lower smooth curves on the left are based on Equations 4 and 5, respectively; the upper and lower smooth curves on the right are based on Equations 6 and 7 , respectively. 
direction. The left panel shows the results obtained with the protractor, and the right panel shows the results obtained with verbal estimation. The two left curves are based on Equations 4 and 5, and the two right curves are based on Equations 6 and 7. It is clear from Figure 8 that the ratio of obtained angle to visual angle decreases as visual angle increases.

Figure 8 may provide a basis for explaining overconstancy of size, or the observation that the apparent size of an object of fixed size increases as the object recedes (Teghtsoonian, 1974). For example, when a far standard is matched by a near variable under the objective-size instructions, the variable is adjusted so that it appears to have a larger physical size than the standard's (Baird, 1970; Epstein, 1963; Smith, 1953). According to the traditional explanation of overconstancy (e.g, Carlson, 1960), the subject believes that the far standard is perceived to be smaller than it actually is, and therefore, to compensate for this reduction in the apparent size of the standard, the subject adjusts the size of the variable so that the variable appears to be larger than the standard.

Our explanation of overconstancy does not necessitate such a cognitive compensation. Instead, it is assumed that visual angle in the apparent-distance theory should be replaced by apparent visual angle. As an illustration, consider the situation in which two objects of the same physical size are viewed at different physical distances $\left(D_{f}\right.$ and $D_{n}$ ), with $D_{f}$ being $k$ times as far away as $D_{n}$. If the visual angles of the two objects $\left(\theta_{f}\right.$ and $\left.\theta_{n}\right)$ are so small that $\tan \theta$ equals $\theta$ approximately, we obtain

$$
\theta_{n} \simeq k \theta_{f}
$$

where $\simeq$ denotes an approximation.

In this situation, when the apparent distance of the far object $\left(D_{f}^{\prime}\right)$ is $k^{\prime}$ times as far as the apparent distance of the near object $\left(D_{n}^{\prime}\right)$, we obtain

$$
D_{f}^{\prime}=k^{\prime} D_{n}^{\prime} \text {. }
$$

When distance is perceived in proportion to actual distance $\left(D_{f}^{\prime} / D_{n}^{\prime}=D_{f} / D_{n}\right)$, we obtain $k^{\prime}=k$. If apparent distance is an accelerating function of actual distance, we obtain $k^{\prime}>^{\prime} k$, whereas if apparent distance is a decelerating function of actual distance, we obtain $k^{\prime}<k$.

The apparent visual angles of the two objects $\left(\theta_{n}^{\prime}\right.$ and $\left.\theta_{f}^{\prime}\right)$ are

$$
\theta_{n}^{\prime}=N \theta_{n} \simeq k N \theta_{f}
$$

and

$$
\theta_{f}^{\prime}=F \theta_{f},
$$

where $N=\theta_{n}^{\prime} / \theta_{n}$ and $F=\theta_{f}^{\prime} / \theta_{f}$. Note that, since $\theta_{n}$ is larger than $\theta_{f}$ in this situation, we have

$$
\frac{F}{N}>1 \text {. }
$$

If apparent visual angle is transformed into apparent size by taking apparent distance into account, it can be obtained from Equations 9, 10, and 11 that the apparent sizes of the near object $\left(S_{n}^{\prime}\right)$ and the far object $\left(S_{f}^{\prime}\right)$ would be

$$
S_{n}^{\prime}=D_{n}^{\prime} \theta_{n}^{\prime}=k N D_{n}^{\prime} \theta_{f}
$$

and

$$
S_{f}^{\prime}=D_{f}^{\prime} \theta_{f}^{\prime}=k^{\prime} F D_{n}^{\prime} \theta_{f}
$$

From Equations 13 and 14, we have

$$
\frac{S_{f}^{\prime}}{S_{n}^{\prime}} \simeq \frac{k^{\prime} F}{k N}
$$

From Equations 12 and 15, it can be seen that overconstancy $\left(S_{f}^{\prime} / S_{n}^{\prime}>1\right)$ occurs when apparent distance is an accelerating function of actual distance $\left(k^{\prime} / k>1\right)$. This prediction is also derived from the apparent-distance theory. Furthermore, it is possible that overconstancy occurs when apparent distance is a linear or decelerating function of actual distance but $k^{\prime} / k$ is larger than $N / F$ (i.e., $\left.N / F<k^{\prime} / k \leq 1\right)$. This prediction is not derived from the apparent-distance theory. Baird and Biersdorf (1967), Epstein (1963), and Higashiyama and Kitano (1991) found an overconstancy effect when distance estimates grew less rapidly than actual distance, in agreement with our prediction.

\section{Apparent Visual Angle of the Moon at Different Elevations}

The moon illusion refers to the relative apparent size of the moon seen at different elevations: The moon appears to be largest at the horizon, and its apparent size decreases as it ascends to higher elevations, appearing smallest at the zenith.

Although there are other explanations of the moon illusion (e.g., Hershenson, 1989), McCready $(1985,1986)$ recently contended that the moon illusion could be accounted for if the apparent visual angle of the moon decreased as the moon ascended. Nevertheless, he did not attempt to measure this apparent visual angle, because its apparent extent is so small $\left(0.5^{\circ}\right)$ that it is very difficult to measure.

From each of Equations 2, 4, and 6, however, it is possible to derive the apparent extent, $M$, of the visual angle $s$ that is situated at elevation $\theta(s<\theta)$ :

$$
M=\theta_{\mathrm{v}}^{\prime}(\theta)-\theta_{\mathrm{v}}^{\prime}(\theta-s) .
$$

This result may be normalized to the visual angle near the horizon, which is given by

$$
M_{c}=\theta_{\mathrm{v}}^{\prime}\left(\theta_{c}\right)-\theta_{\mathrm{v}}^{\prime}\left(\theta_{c}-s\right),
$$

where $\theta_{c}=1^{\circ}$.

Figure 9 shows $M / M_{c}$ as a function of $\theta$, with $s=0.5^{\circ}$. The three curves in Figure 9 were obtained from Equations 2, 4, and 6. The curves from Equations 2 and 4 are very similar to one another, but they are both different from the curve from Equation 6 . This difference reflects the differences between the quadratic coefficients; the smaller the quadratic coefficient, the more $M / M_{c}$ ap- 


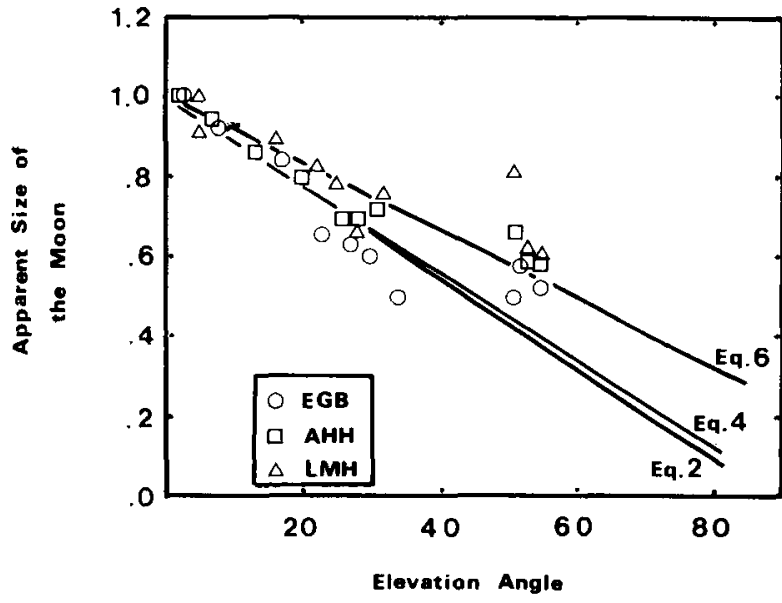

Figure 9. The ratio of apparent size of the moon at different elevations (in degrees) to the apparent size of the borizontal moon. The parameter is the data from the 3 subjects in Holway and Boring (1940). The three smooth lines are predicted from Equations 2, 4 , and 6.

proaches unity, implying the disappearance of the moon illusion. $^{5}$

To examine how well these curves explain the moon illusion, a classic set of data taken from Holway and Boring (1940) is plotted in Figure 9. In Holway and Boring's experiment, 3 subjects (E.G.B., A.H.H., and L.M.H.) adjusted a variable near target so that its apparent size equaled the apparent size of the moon at different elevations as seen from the top of a building. For comparison with our prediction, we normalized the variable sizes obtained from each subject relative to the variable size matched for a horizontal moon.

The degree of fit may not be perfect, but the trend that the moon appears smaller as it ascends is largely explainable in terms of the moon's apparent visual angle. This also suggests that information about the apparent distance to the moon is not indispensible to the visual system as it determines the moon's apparent size.

We generally think that the importance of apparent distance should not be disregarded when we consider the perception of size. The present study, however, suggests that for extremely distant objects such as the moon, apparent distance is not as critical as apparent visual angle is. In fact, asking the subjects to estimate the apparent distance to the sky, Baird and Wagner (1982) did not find evidence that they perceived the sky as a localized surface.

\section{Ecological Significance of Enlarging Visual Angle}

From an ecological point of view, the tendency to magnify visual angle seems to be of benefit in real life. If we were to perceive a visual angle as it actually is, it might be difficult to read small letters, thread a needle, pick up something with tweezers, and so forth. The visual system resembles a convex lens that enlarges objects and pictures and helps people who experience difficulty in read- ing due to presbyopia. However, the visual system is not exactly the same as the convex lens in every respect. An ordinary convex lens has a fixed magnification, so that any object seen through it is enlarged according to a constant. On the other hand, the visual system seems to have a flexible magnification that decreases as visual angle increases. This property may be ecologically valid, because it is not necessary to enlarge a large visual angle.

\section{REFERENCES}

Avery, G. C., DAY, R. H. (1969). Basis of the horizontal-vertical illusion. Journal of Experimental Psychology, 81, 376-380.

BAIRD, J. C. (1970). Psychophysical analysis of visual space. Oxford: Pergamon.

BAird, J. C., Biersporf, W. R. (1967). Quantitative functions for size and distance judgments. Perception \& Psychophysics, 2, 161-166.

BaIRD, J. C., W WGNer, M. (1982). The moon illusion: I. How high is the sky? Joumal of Experimental Psychology: General, 111, 296-303.

Begelman, D. A., Steinfeld, G. (1967). An investigation of several parameters of the horizontal-vertical illusion. Perception \& Psychophysics, 2, 539-543.

CarLSON, V. R. (1960). Overestimation in size-constancy judgments. American Journal of Psychology, 73, 199-213.

Chapanis, A., Mankin, D. A. (1967). The vertical-horizontal illusion in a visually-rich environment. Perception \& Psychophysics, 2, 249-255.

Dick, M., Hochstein, S. (1989). Visual orientation estimation. Perception \& Psychophysics, 46, 227-234.

EPSTEIN, W. (1963). Attitude of judgment and the size-distance invariance hypothesis. Joumal of Experimental Psychology, 66, 78-83.

FoLeY, J. M. (1965). Visual space: A scale of perceived relative direction. Proceedings of the 73rd Annual Convention of the American Psychological Association, 1, 49-50.

FoLEY, J. M., \& HELD, R. (1972). Visually directed pointing as a function of target distance, direction, and available cues. Perception \& Psychophysics, 12, 263-268.

Giunsky, A. S. (1955). The effect of attitude on the perception of size. American Journal of Psychology, 68, 173-192.

GoGel, W. C. (1973a). The organization of perceived space: I. Perceptual interactions. Psychologische Forschung, 36, 195-221.

Gogel, W. C. (1973b). The organization of perceived space: II. Consequences of perceptual interactions. Psychologische Forschung, 36, 223-247.

Hagen, M. A., Jones R. K., \& Reed, E. S. (1978). On a neglected variable in theories of pictorial perception: Truncation of the visual field. Perception \& Psychophysics, 23, 326-330.

Hershenson, M. (1989). The moon illusion, Hillsdale, NJ: Erlbaum.

Higashiyama, A. (1984). Curvature of binocular visual space: A modified method of right triangle. Vision Research, 24, 1713-1718.

HigashiYama, A. (1987). Ryogan-shi-kukan to fukuso no kino [Role of convergence in binocular space perception]. Japanese Psychological Monographs, No. 18.

Higashiyama, A. (1991). The horizontal-vertical illusion in outdoor settings. Unpublished manuscript.

Higashiyama, A., Kitano, S. (1991). Perceived size and distance of persons in natural outdoor settings: The effects of familiar size. Psychologia, 34, 188-199.

Holway, A. H., Boring, E. G. (1940). The moon jllusion and the angle of regard. American Joumal of Psychology, 53, $109-116$.

Holway, A. H., Boring, E. G. (1941). Determinants of apparent visual size with distance variant. American Journal of Psychology, 54, 21-37.

Kammann, R. (1967). The overestimation of vertical distance and slope and its role in the moon illusion. Perception \& Psychophysics, 2, 585-589. 
KÜNNAPAS, T. M. (1955). An analysis of the "vertical-horizontal illusion." Journal of Experimental Psychology, 49, 134-140.

LENNIE, P. (1971). Distortions of perceived orientation. Nature New Biology, 233, 155-156.

MCCreAdy, D. (1985). On size, distance, and visual angle perception. Perception \& Psychophysics, 37, 323-334.

McCready, D. (1986). Moon illusions redescribed. Perception \& Psychophysics, 39, 64-72.

Morinaga, S., Noguchi, K., OHISHI, A. (1962). The horizontalvertical illusion and the relation of spatial and retinal orientation. Japanese Psychological Research, 4, 25-29.

MYERS, J. L. (1966). Fundamentals of experimental design (3rd ed.). Boston: Allyn \& Bacon.

NaKATANI, K. (1991). Katamuki no keiji handan oyobi zettai handan ni okeru oblique koka to kategori teki yoin [Categorical factors in the oblique effect under successive and absolute judgments of inclination] Japanese Journal of Psychology, 61, 400-403.

Ogasawara, J. (1933). Shi-chikaku ni okeru ookisa no kojosei ni tsuite [Size constancy in visual perception]. Japanese Journal of Psychology, 8, 549-577.

OHNO, S. (1972). Chikaku kukan no ihosei [Anisotropy of perceptual space]. In Y. Akishige (Ed.), Chikaku-teki sekai no kojosei: Ninshiki shinrigaku [Constancies of the perceptual world: Epistemological psychology] (Vol. 4, pp. 387-575). Tokyo: Ibunsha.

Rock, 1. (1975). An introduction to perception. New York: Macmillan.

Rosenthal, R., \& Rosnow, R. (1985). Contrast analysis: Focused comparisons in the analysis of variance. Cambridge: Cambridge University Press.

Ross, H. E. (1974). Behaviour and perception in strange environments. London: Allen \& Unwin.

Smith, W. M. (1953). A methodological study of size-distance perception. Journal of Psychology, 35, 143-153.

Teghtsoonian, M. (1974). The doubtful phenomenon of over-constancy. In H. R. Moskowitz, B. Scharf, \& J. C. Stevens (Eds.), Sensation and measurement: Papers in honor of S. S. Stevens (pp. 411-420). Dordrecht: D. Reidel.

\section{NOTES}

1. Even though the lower end of the vertical standard was specified with the red mark, the possibility might have remained that some subjects mistook the lower end for the reference ground $(O)$. If such a confusion took place, the protractor would sometimes have been adjusted to more than $90^{\circ}$, because the visual angle between the roof and the ground was $102.2^{\circ}$ for a viewing distance of $3 \mathrm{~m}$. But no subject provided an obtuse angle.

2. One might argue that when Equation 1 is fitted to matched angles, the constant coefficient $a$ should be assumed to be zero, because we receive no information of angle for the visual angle of $0^{\circ}$. Yet although this suggestion may seem intuitively correct, it is not always correct in practice when the data are described (see also note 3 ). The problem as to whether the constant coefficient equals zero or not, therefore, ought to be determined by a statistical test. If the constant coefficient is not zero, we obtain $\theta^{\prime}=a$ for the visual angle of $0^{\circ}$. This outcome does not mean that the visual angle of $0^{\circ}$ would produce an apparent angle of $a^{\circ}$. In this case, the angle of $a^{\circ}$ may be interpreted as a bias that intrudes into angle judgments. This bias is produced consistently, independently of visual angle. Note also that when Equation 1 is fitted to the data, the obtained function should not be used to predict matches (or estimates) for extremely small and large visual angles $\left(\theta \leq 3^{\circ}\right.$ and $\theta \geq 80^{\circ}$ ). The predictions should be limited to the angle range employed in each experiment.

3. One might argue that when the linear function is fitted to matched angles, we should assume the intercept of zero. However, it is difficult to make such an assumption for all subjects. Figure 10 shows matches obtained from 3 of the subjects in Experiment 2. It appears from Figure 10 that the linear functions for Subjects 1 and 2 do not go through the origin, whereas the linear functions for Subject 3 may go through the origin. Table 3 enables us to compare the slopes, intercepts, and unbiased standard errors of estimates $\left(\sigma_{y x}\right)$ of linear functions through the origin with those of the standard linear functions with two free parameters. For example, the $\sigma_{y x}$ of Subject 1 for the vertical direction is $4.69^{\circ}$ for the standard linear function, but $9.82^{\circ}$ for the linear function through the origin. Needless to say, the linear function through the origin does not provide a good fit for Subject 1's data.

When the linear function-assuming an intercept of zero-does not provide a good fit, one may attempt to use other models that go through the origin. It appears to us that this claim is based on the assumption that our methods of measurement (i.e., methods of angle production, distance production, and verbal estimation) provide pure apparent visual angle that is not contaminated by a judgmental bias and that, therefore, apparent visual angle would be zero for a visual angle of $0^{\circ}$. This assumption seems too strong not only for our data but for other metric properties of visual space. Hagen, Jones, and Reed (1978) described matched size (and distance) as a linear function of actual size (and distance). The obtained intercepts were positive for all conditions employed. Foley and Held (1972) found that pointed distance of a visual target was a linear function of target distance, and that both the slope and the intercept varied with visual direction and available distance cues.

4. We also calculated the ratio of vertical to horizontal matches (and estimates) on the basis of the power functions. Since, from the method with a protractor, $\theta_{v}^{\prime}=4.80 \theta^{0.639}$ for the vertical and $\theta_{\mathrm{h}}^{\prime}=3.50 \theta^{0.701}$ for the horizontal, $\theta_{\mathrm{v}}^{\prime} / \theta_{\mathrm{h}}^{\prime}=1.37 \theta^{-0.062}$. This means that the $\theta_{\mathrm{v}}^{\prime} / \theta_{\mathrm{h}}^{\prime}$ decreases monotonically with visual angle. For example, for the visual angles of $1^{\circ}, 5^{\circ}, 10^{\circ}, 20^{\circ}, 40$, and $80^{\circ}, \theta_{v}^{\prime} / \theta_{h}^{\prime}$ equals $1.37,1.24,1.19,1.13,1.09$,

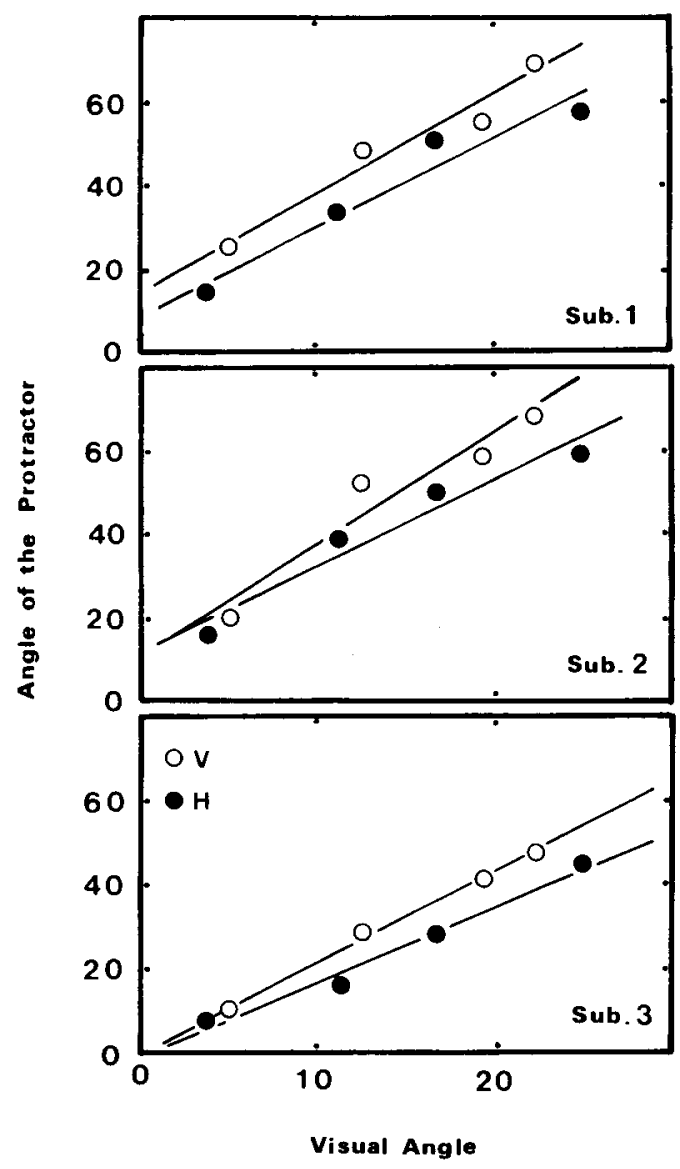

Figure 10. The results from 3 of the subjects in Experiment 2. Matched angle of the protractor (in degrees) is shown as a function of visual angle (in degrees). Open circles stand for the vertical angle; filled circles stand for the horizontal angle. 
Table 3

Slopes, Intercepts, and Unbiased Standard Errors of Estimates $\sigma_{y x}$ of Two Linear Functions Fitted to Matched Angles for each of Three Subjects

\begin{tabular}{|c|c|c|c|c|c|c|}
\hline \multirow{2}{*}{$\begin{array}{c}\text { Linear } \\
\text { Function }\end{array}$} & \multicolumn{3}{|c|}{ Vertical } & \multicolumn{3}{|c|}{ Horizontal } \\
\hline & Slope & Intercept & $\sigma_{y x}$ & Slope & Intercept & $\sigma_{y x}$ \\
\hline \multicolumn{7}{|c|}{ Subject 1} \\
\hline$\theta^{\prime}=$ & & & & & & \\
\hline$a+b \theta$ & 2.32 & 14.97 & 4.69 & 2.11 & 9.25 & 5.76 \\
\hline$\theta^{\prime}=a \theta$ & 3.16 & & 9.82 & 2.62 & & 8.50 \\
\hline \multicolumn{7}{|c|}{ Subject 2} \\
\hline$\theta^{\prime}=$ & & & & & & \\
\hline$a+b \theta$ & 2.67 & 10.03 & 6.97 & 2.03 & 12.32 & 5.04 \\
\hline$\theta^{\prime}=a \theta$ & 3.24 & & 9.05 & 2.70 & & 9.77 \\
\hline \multicolumn{7}{|c|}{ Subject 3} \\
\hline$\theta^{\prime}=$ & & & & & & \\
\hline $\begin{array}{l}a+b \theta \\
\theta^{\prime}=a \theta\end{array}$ & $\begin{array}{l}2.17 \\
2.15\end{array}$ & -0.25 & $\begin{array}{l}1.24 \\
1.25\end{array}$ & $\begin{array}{l}1.78 \\
1.71\end{array}$ & -1.27 & $\begin{array}{l}2.64 \\
2.78\end{array}$ \\
\hline
\end{tabular}

and 1.05 , respectively. By the method of verbal estimation, $\theta_{v}^{\prime}=$ $3.67 \theta^{0.713}$ for the vertical, and $\theta_{\mathrm{h}}^{\prime}=3.29 \theta^{0.703}$ for the horizontal. Therefore, $\theta_{\mathrm{v}}^{\prime} / \theta_{\mathrm{h}}^{\prime}=1.12 \theta^{0.010}$. This means that $\theta_{\mathrm{v}}^{\prime} / \theta_{\mathrm{h}}^{\prime}$ increases gradually with visual angle. For example, for the visual angles of $1^{\circ}, 5^{\circ}, 10^{\circ}, 20^{\circ}$, $40^{\circ}$, and $80^{\circ}, \theta_{v}^{\prime} / \theta_{\mathrm{h}}^{\prime}$ equals $1.12,1.13,1.14,1.15,1.16$, and 1.17 , respectively. It is thus clear that the function $\theta_{\mathrm{v}}^{\prime} / \theta_{\mathrm{h}}^{\prime}=f(\theta)$ that is determined by the power function varies with the method of measurement. This suggests that the power function does not provide as reliable a value of $\theta_{\mathrm{v}}^{\prime} / \theta_{\mathrm{h}}^{\prime}$ as does the quadratic function.
Despite this suggestion, one may discuss that the different trends of the function $\theta_{v}^{\prime} / \theta_{\mathrm{h}}^{\prime}=f(\theta)$ reflect the intrinsic differences between the data obtained with the two methods of measurement, rather than a defect of the power function. However, the two sets of data shown in Figures 4 and 6 do not seem greatly different. Both figures show that the differences between vertical and horizontal matches (and estimates) are smallest at small angles and largest at medium angles. These trends are represented well in Figure 7, which was drawn on the basis of the quadratic function.

5. From Equation 16, we obtain

$$
\begin{aligned}
M & =\left(a+b \theta-c \theta^{2}\right)-\left[a+b(\theta-s)+c(\theta-s)^{2}\right] \\
& =s(b+2 c \theta-c s) .
\end{aligned}
$$

From Equation 17, we obtain

$$
M_{c}=s\left(b+2 c \theta_{c}-c s\right) .
$$

From Equations 18 and 19 , we obtain

$$
\frac{M}{M_{c}}=\frac{b-c s}{b+c\left(2 \theta_{c}-s\right)}+\frac{2 c}{b+c\left(2 \theta_{c}-s\right)} \theta .
$$

From Equation 20, it is clear that $M / M_{c}$ is a linear function of $\theta$ with a slope of $2 c /\left[b+c\left(2 \theta_{c}-s\right)\right]$ and an intercept of $(b-c s) /\left[b+c\left(2 \theta_{c}-s\right)\right]$. Thus, the slope, which is closely related to the moon illusion, is determined by both the linear and the quadratic coefficients, and it is free from the constant coefficient. However, the quadratic coefficient may be more critical than the linear coefficient, because for a zero value of $c, M / M_{c}=1$, and for a negative (positive) value of $c, M / M_{c}$ is a negative (positive) linear function of $\theta$. (Note that $b+c\left[2 \theta_{c}-s\right]>0$.)

(Manuscript received October 10, 1990; revision accepted for publication October 1, 1991.) 